\title{
No Significant Difference in Outcomes for Surgical Treat- ment of Thyroid Cancer in Kidney Transplant Recipients - Analysis of the HCUP NIS Data
}

\author{
Darren B Abbas, MD*, Eric Goldschmidt, MD, Christina Camick, MD, Weikai Qu, MD, \\ PhD and Jorge Ortiz, MD
}

Department of Surgery, The University of Toledo, USA

*Corresponding author: Darren B Abbas, MD, Clinical Instructor of Surgery, University of Toledo College of Medicine and Life Sciences, Department of Surgery - 3000 Arlington Ave. Toledo, OH 43614, USA, Tel: (214)-500-6352, Fax: (419)-3833348

\begin{abstract}
Solid organ transplant recipients, and in particular Kidney Transplant (KTx) patients, are at increased risk for developing thyroid cancers due to immunosuppression and improved long-term survival post-transplant. Using the HCUP NIS database, we compared mortality rate, disposition, LOS, and total hospital charge between KTx and Non-KTx patients following thyroidectomy for thyroid cancer during 2008-2014. Propensity score matching was performed using demographics, payer, income, hospital characteristics, elective admission, and in-hospital mortality index score. There were no significant differences in mortality rate, disposition, LOS, and total hospital charge between KTx and Non-KTx patients following both univariable analysis and propensity score matching even though KTx patients have a significantly higher number of chronic conditions and in-hospital mortality index score. Our data shows that KTx patients can safely undergo thyroidectomy for cancer despite being a high-risk population.
\end{abstract}

\section{Keywords}

Thyroidectomy, Kidney transplant, Thyroid cancer, Papillary thyroid cancer, Social determinants, Outcomes, Cost analysis

\section{Introduction}

The overall incidence of thyroid cancer has risen substantially in recent years. In the United States, the incidence has increased roughly $211 \%$ since 1975 with an estimated 63,000 new cases in 2014 alone [1-4]. It is one of the most common cancers diagnosed today.
Kidney transplant recipients (KTXR) are a population at increased risk for developing a number of malignancies, including Thyroid Cancer (TC), due to chronic immunosuppression [5-11]. A number of single-center and population-based studies have identified KTXR among the highest risk of all solid organ transplant recipients to develop TC, typically papillary thyroid cancer, at a relative risk ranging from 2.2-9.5. Furthermore, this population also presents with more advanced disease requiring lymph node dissection for surgical treatment [12].

As the annual number of kidney transplants performed increases and long-term survival of these transplant recipients improves with better immunosuppression and perioperative care, KTxR are more frequently encountered within general surgery and its subspecialties $[13,14]$. Solid organ transplant recipients who undergo emergency abdominal surgeries experienced worse outcomes when compared to non-transplant patients with morbidity and mortality rates estimated at nearly $33 \%$ and $18 \%$, respectively, compared to $9 \%$ and $0.5 \%$ [15]. This discrepancy was theorized to stem from a combination of immunosuppression and comorbidities related to the effects of chronic kidney disease existing prior to transplantation [16].

Several recent follow-up studies demonstrated worse outcomes after operations such as cholecys- 
tectomy, appendectomy, and colorectal resection in transplant recipients. The outcomes measured included overall complications, mortality, Length of Stay (LOS), and cost of stay at both transplant and nontransplant centers [17-19].

As the incidence of thyroid cancer increases in conjunction with the increasing number of KTxR in the general population, a better understanding of outcomes after thyroidectomy in these complex patients is warranted. To expand on existing literature, we utilized the National Inpatient Sample (NIS) database to investigate differences in morbidity, mortality, length of stay, and cost between an adult population of nonKTxR and KTxR undergoing thyroidectomy for thyroid cancer from 2008-2014.

\section{Methods and Materials}

Subjects were identified from hospital data available through the Health Care Utilization Project Nationwide Inpatient Sample (HCUP-NIS) database from 2008-2014. The National Inpatient Sample (NIS) contains all-payer data on hospital stays from states participating in the Healthcare Cost and Utilization Project (HCUP) and includes over 7 million hospital stays per year. The NIS approximates a $20 \%$ stratified sample of discharges from United States hospitals and provides clinical and resource use information included in a typical discharge abstract [20].

We included adults ( $\geq 18$-years-old) who had International Classification of Disease, Ninth Revision

Raw Treated

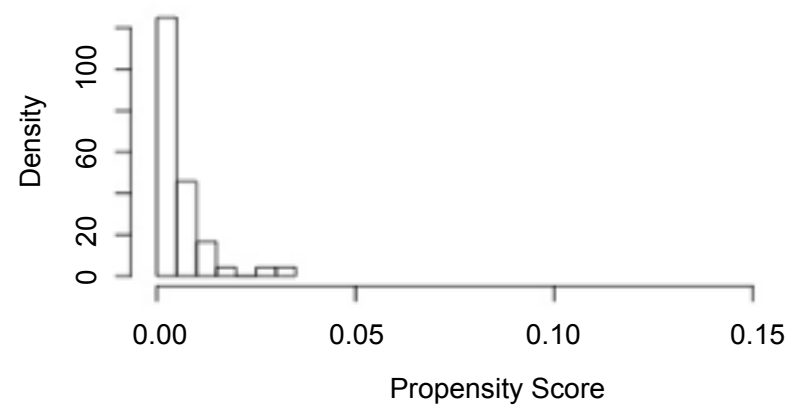

Raw Control

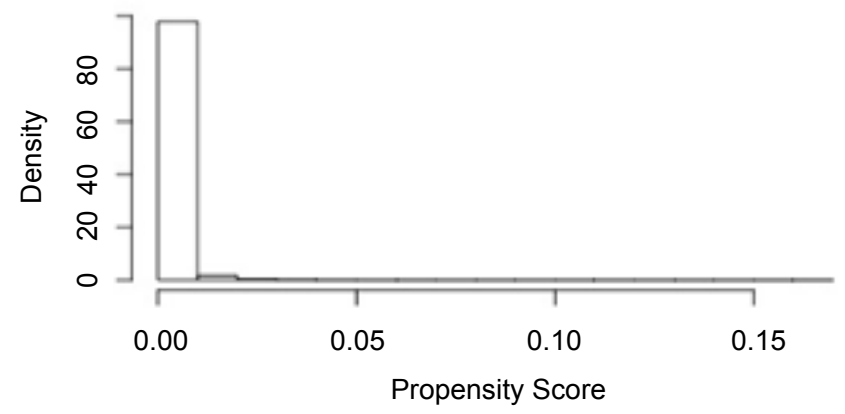

(ICD-9) diagnosis codes for thyroid cancer and who also underwent ICD-9 procedure codes for thyroidectomy (unilateral thyroid lobectomy, another partial thyroidectomy, total thyroidectomy, and substernal thyroidectomy). From this search, subjects were divided into two cohorts based on a history of kidney transplant (KTxR) prior to thyroidectomy, as identified by ICD-9 procedure code, or no history of kidney transplant (non-KTxR). Demographics, hospital characteristics, insurance, household income, and AHRQ in-hospital mortality score were compared.

Propensity score matching was performed using $\mathrm{R}$ statistical software and the Matchlt package to find matching cohorts for comparison between KTxR and Non-KTxR. Factors used for calculation of propensity score included demographics, primary payer, household income, hospital characteristics, elective admission, and In-Hospital Mortality Index Score (IHMIS). A greedy nearest neighbor matching method with a $2: 1$ ratio was implemented to select the best subgroup of patients from the control thyroidectomy group that matched the transplant recipients. Matched data was then exported into IBM SPSS software for further statistical analysis.

Continuous data were presented as either mean \pm SD or median/IQR; Student's T-test or Mann-Whitney U test (continuous DVs) compared differences depending on whether the data was normally distributed. Categorical data were presented as rate/ratio and Chi-square test compared the different groups. IBM SPSS statistics
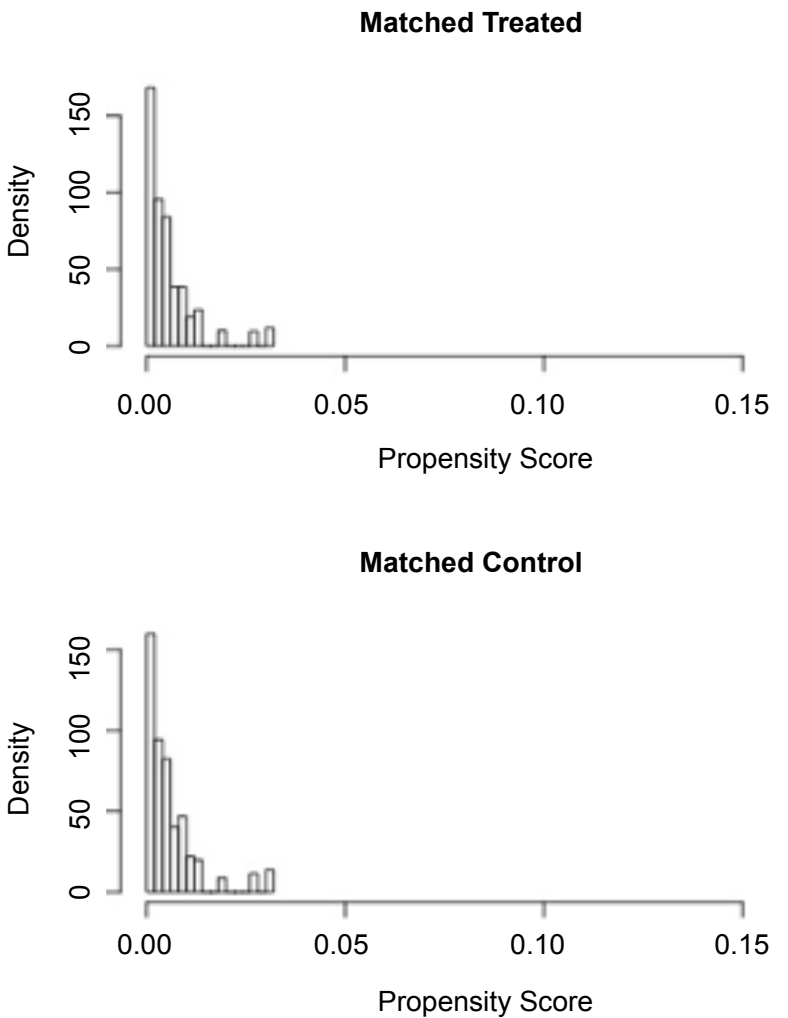

Figure 1: Histogram of Propensity Score in Raw Data and Matched Data. 
ver. 24 (IBM Corp., Armonk, NY) performed all statistical analyses. Type I error level was set at 0.05 . All analyses were 2-sided.

Indices of outcome compared between the KTX and non-KTX cohorts included: in-hospital mortality (MR), Length of Stay (LOS), Total Hospital Charge (TOTCHG), and complications which were defined by specific ICD9 diagnosis codes including hemorrhage, hematoma, seroma, post-operative infection, disruption of surgical wound, injury of parathyroid, and injury of recurrent laryngeal nerve.

\section{Results}

We estimated that 126,675 people with thyroid cancer underwent thyroidectomy during 2008-2014 from NIS data. KTxR accounted for $0.2 \%(n=255)$. 26,666 of the 126,675 met our inclusion criteria, yielding a total of $53 \mathrm{KTXR}$ who underwent thyroidectomy for thyroid cancer during this time period with these inclusion criteria. Using propensity scoring as described above, a control cohort of non-KTx thyroidectomy subjects $(n=96)$ was obtained to compare to our KTxR cohort $(n=53)$. A histogram of propensity scoring between the KTxR and non-KTxR groups before and after matching is shown in Figure 1.

Given that the majority of case numbers in the
KTxR group were fewer than 10 , our data is presented in percentages to comply with HCUP regulations. Comparison of demographics between the two cohorts is shown in Table 1. There was no significant difference in age $(53.4 \%$ vs. $51.5 \%, p=0.272)$. The KTXR cohort had a significantly higher percentage of males (43.4\% vs. $26.9 \%, p=0.007)$ and Native Americans $(4.0 \%$ vs. $0.4 \%, p=<0.001)$. Table 2 shows that the KTXR cohort had a significantly higher percentage of subjects with Medicare insurance $(50.9 \%$ vs. $23.6 \%$, $p=<0.001)$ and a significantly lower percentage of subjects with private insurance $(47.2 \%$ vs. $61.4 \%, p=$ $0.033)$. There was no significant difference in median household income level.

The characteristics of the hospitals in which both cohorts underwent thyroidectomy were compared (Table 3 and Table 4). There was no significant difference identified in size, ownership, location, teaching status, or region of hospital. The majority in both groups received care at hospitals classified as large $(73.6 \%$ and $69.5 \%)$, private, non-profit (79.2\% and $78.2 \%)$, and urban, teaching (67.3\% and $66 \%)$.

Table 5 demonstrates KTxR had a significantly higher number of chronic conditions ( 6 vs. $3, p=<0.001$ ) and a higher in-hospital mortality index score ( 5 vs. $0, p=<$ 0.001).

Table 1: Comparison of Demographics between Kidney Transplant and Non-Kidney Transplant Recipients.

\begin{tabular}{|l|l|l|l|l|}
\hline & Overall & KTx (\%) & Non-KTx (\%) & P \\
\hline Age (years, Mean +/- SD) & $51.5+/-15.7$ & $53.4+/-12.8$ & $51.5+/-15.7$ & 0.272 \\
\hline Male & 26.9 & 43.4 & 26.9 & 0.007 \\
\hline Race & & & & 0.004 \\
\hline White & 69.9 & 62.0 & 70.0 & \\
\hline African American & 7.1 & 8.0 & 7.1 & 12.4 \\
\hline Hispanic & 12.4 & 14.0 & 5.9 & \\
\hline Asian/Pacific Islander & 5.9 & 4.0 & 0.4 & $<0.001$ \\
\hline Native American & 0.4 & 4.0 & 4.3 & \\
\hline Other & 4.3 & 8.0 & \\
\hline
\end{tabular}

Table 2: Differences of Insurance Coverage between Kidney Transplant and Non-Kidney Transplant Recipients.

\begin{tabular}{|l|l|l|l|l|}
\hline & Overall & KTx (\%) & Non-KTx (\%) & P \\
\hline Primary payer & & & & $<0.001$ \\
\hline Medicare & 23.6 & 50.9 & 23.6 & $<0.001$ \\
\hline Medicaid & 8.9 & 1.9 & 8.9 & 0.033 \\
\hline Private & 61.4 & 47.2 & 61.4 & 2.5 \\
\hline Self-Pay & 2.5 & 0.0 & 0.5 & \\
\hline No Charge & 0.5 & 0.0 & 3.2 & 0.240 \\
\hline Other & 3.2 & 0.0 & & \\
\hline Median Household Income & & & 19.1 & \\
\hline $0-25^{\text {th }}$ Quartile & 19.1 & 28.8 & 22.7 \\
\hline $26^{\text {th }}-50^{\text {th }}$ Quartile & 22.7 & 25.0 & 24.8 \\
\hline $5^{\text {st }-75^{\text {th }} \text { Quartile }}$ & 24.8 & 17.3 & 33.4 \\
\hline $76^{\text {th }}-100^{\text {th }}$ Quartile & 33.3 & 28.8 & \\
\hline
\end{tabular}


Table 3: Hospital Size/Ownership of Kidney Transplant and Non-Kidney Transplant Recipients.

\begin{tabular}{|l|l|l|l|}
\hline & Overall & KTx (\%) & Non-KTx (\%) \\
\hline Hospital Ownership & & & 0.730 \\
\hline Government & 13.3 & 15.1 & 13.3 \\
\hline Private, Non-Profit & 78.2 & 79.2 & 78.2 \\
\hline Private, Invest-Own & 8.5 & 5.7 & 8.5 \\
\hline Hospital Size & & & 0.606 \\
\hline Small & 12.0 & 7.5 & 12.0 \\
\hline Medium & 18.5 & 18.9 & 18.5 \\
\hline Large & 69.6 & 73.6 & 69.5 \\
\hline
\end{tabular}

Table 4: Hospital Location of Kidney Transplant and Non-Kidney Transplant Recipients.

\begin{tabular}{|c|c|c|c|c|}
\hline & Overall & KTx (\%) & Non-KTx (\%) & $\mathbf{P}$ \\
\hline Hospital Location/Teaching & & & & 0.731 \\
\hline Rural & 5.2 & 7.5 & 5.2 & \\
\hline Urban, Non-Teaching & 27.6 & 26.4 & 27.6 & \\
\hline Urban, Teaching & 67.3 & 66.0 & 67.3 & \\
\hline Hospital Region & & & & 0.192 \\
\hline Northeast & 33.4 & 28.3 & 33.5 & \\
\hline Midwest & 17.0 & 9.4 & 17.0 & \\
\hline South & 24.4 & 26.4 & 24.4 & \\
\hline West & 25.1 & 35.8 & 25.1 & \\
\hline
\end{tabular}

Table 5: Comparison of Chronic Conditions and Mortality Index Score between Kidney Transplant and Non-Kidney Transplant Recipients.

\begin{tabular}{|l|l|l|l|l|}
\hline & Overall & KTx & Non-KTx & P \\
\hline Number of Chronic Conditions (median/IQR) & $3(2-5)$ & $6(4.5-7)$ & $3(2-5)$ & $<0.001$ \\
\hline In-hospital Mortality Index Score (Median/IQR) & $0(-1-8)$ & $5(5-14)$ & $0(-1-8)$ & $<0.001$ \\
\hline Elective Admission (\%) & 10 & 7.5 & 10 & 0.555 \\
\hline
\end{tabular}

Table 6: Incidence of Post-Operative Complications of Kidney Transplant vs. Non-Kidney Transplant Recipients.

\begin{tabular}{|l|l|l|l|l|}
\hline & Overall & KTx (\%) & Non-KTx (\%) & p \\
\hline Postop Infection & 0.2 & 0.0 & 0.2 & 0.770 \\
\hline Postop hemorrhage/hematoma/seroma & 1.7 & 0.0 & 1.7 & 0.334 \\
\hline Disruption of wound & 0.1 & 0.0 & 0.1 & 0.862 \\
\hline Hypoparathyroidism & 1.8 & 0.0 & 1.8 & 0.327 \\
\hline Paralysis of vocal cords & 2.6 & 0.0 & 2.6 & 0.232 \\
\hline
\end{tabular}

Table 7: Hospital Stay Metrics Before Propensity Score Matching of Kidney Transplant and Non-Kidney Transplant Recipients.

\begin{tabular}{|l|l|l|l|l|}
\hline & Overall & KTx (\%) & Non-KTx (\%) & P \\
\hline LOS (days, median/IQR) & $1(1-2)$ & $1(1-2)$ & $1(1-2)$ & 0.398 \\
\hline TOTCHG (US\$, median/IQR) & $25,885(17,428-40,054)$ & $27,607(17,583-36,752.2)$ & $25,883(17,428-40,058)$ & 0.761 \\
\hline Died & 0.2 & 0.0 & 0.2 & 0.765 \\
\hline Disposition of Patients & & & & 0.984 \\
\hline Routine Discharge & 95.6 & 98.1 & 95.6 & 0.1 \\
\hline STH & 0.1 & 0.0 & 1.3 & \\
\hline SNF/ICF/Other & 1.2 & 0.0 & 2.9 & \\
\hline HHC & 2.9 & 1.9 & \\
\hline
\end{tabular}

The incidence rates of post-operative complications following thyroidectomy were low for both KTXR and
non-KTxR, with no post-operative complications found in the KTxR group (Table 6). Overall, there was no sig- 
Table 8: Hospital Stay Metrics After Propensity Score Matching of Kidney Transplant and Non-Kidney Transplant Recipients.

\begin{tabular}{|l|l|l|l|l|}
\hline & Overall & KTx (\%) & Non-KTx (\%) & P \\
\hline LOS (days, median/IQR) & $2(1-3)$ & $1(1-2)$ & $2(1-3)$ & 0.246 \\
\hline TOTCHG (US\$, median/IQR) & $28,358(18,761.5-$ & $\begin{array}{l}27,607(17,386.25 \\
-36,752.5)\end{array}$ & $\begin{array}{l}29,793(20,363.75- \\
56,208)\end{array}$ & 0.170 \\
\hline Died & $47,766.2)$ & 0.0 & 1.0 & 0.478 \\
\hline Disposition of Patients & 0.7 & & & 0.402 \\
\hline Routine Discharge & & 97.9 & 90.6 & 3.1 \\
\hline SNF/ICF/Other & 93.1 & 0.0 & 5.2 & \\
\hline HHC & 2.1 & 2.1 & & \\
\hline
\end{tabular}

nificant difference in incidence of rate of postoperative complications between the KTXR and non-KTxR.

We compared mortality rate, disposition upon discharge, length of stay, and total hospital charge between KTxR and non-KTxR in a univariable analysis before matching (Table 7) and after propensity score matching (Table 8). There was no significant difference in these outcomes before or after propensity score matching. The average length of hospital stay was 1-2 days for KTXR and 1-3 days for the propensity score matched non-KTXR. The majority both groups were discharged to home $(97.9 \%$ and $90.6 \%)$.

\section{Discussion}

In this national, retrospective matched cohort study of 26,613 non-kidney transplant and 53 kidney transplant patients undergoing thyroidectomy for thyroid cancer, the KTXR had no statistically significant difference in incidence rate of postoperative complications, 30-day mortality rate, disposition, hospital characteristics, length of stay, and total hospital charge both before and after propensity score matching. Our statistical analysis and data are based on reports by DeBrito, et al. measuring outcomes and cost of transplant recipients compared to non-transplant patients undergoing general surgical procedures such as cholecystectomy, appendectomy, and colorectal resections using the HCUP NIS database [17-19].

There was a significant difference in the predominance of males ( $p=0.007)$ and Native Americans $(p<$ 0.001 ) in KTxR compared to the non-KTxR. Multiple reports demonstrate worse outcomes in underrepresented minorities such as Native Americans in healthcare perioperatively [21,22]. However, our data indicate that, despite having a significantly higher proportion of Native Americans, KTxR postoperative complication and mortality rates were equivalent to non-transplant recipients. KTxR also had a significantly higher number of chronic conditions and in-hospital mortality index score, though no differences in 30-day mortality rate. Since KTXR were comprised of a significantly higher proportion of Native Americans, the higher number of chronic conditions would be consistent with current literature that demonstrates that Native Americans are predisposed to chronic conditions such as diabetes and cardiovascular disease at a higher rate than the average non-Hispanic white population [23].

A significantly higher percentage of KTxR possessed Medicare coverage compared to private insurance companies $(p<0.001)$. James, et al. reported that government-subsidized insurance expansion was associated with increased access to the surgical management of thyroid cancer [24]. With federal regulations mandating that Medicare cover patients of any age with end stage renal disease requiring dialysis or kidney transplant, KTxR should also have increased access to surgical management of thyroid cancer. Access to surgical care for thyroid cancer will help spare KTxR from requiring chemotherapy and/or radiotherapy for management of their thyroid cancer, which would decrease the lifespan of their graft and overall survival. Unfortunately, the data from the HCUP NIS database prevents further investigation into the exact timing post-transplant when the KTxR were diagnosed and treated for thyroid cancer.

Kalyoussef, et al. reported that elderly patients endure worse outcomes when undergoing thyroidectomies [25]. Though our data demonstrated no difference in both age and outcomes between the $\mathrm{KTXR}$ and non-KTxR undergoing thyroidectomies, as transplant recipients' lifespans increase with improved immunosuppression regimens, the incidence of thyroid cancer in KTXR should also increase proportionally. Therefore, KTxR will be found to have thyroid cancer at an older age and, with government-subsidized insurance providing greater access to surgical treatment for thyroid cancer as stated above, this population will likely be treated with operative interventions. Further research to determine whether this subset of the population has better, or worse outcomes compared to younger KTXR undergoing thyroidectomies will be of great interest.

As the cost of healthcare continually increases, it is important to note that, though not statistically significant, KTXR had a total hospital charge of $\sim 2000$ more compared to the non-transplant group. This cost difference is likely related to the high cost of the transplant recipients' immunosuppression regimen, but this is just speculation as the HCUP NIS database does not allow us 
to control for this variable. The database does not track costs related to re-admission rates and long-term outcomes/mortalities, so the true lifetime cost associated with thyroidectomies in non-KTxR and KTxR is unable to be calculated with this data. Further investigation into how to minimize the cost of these medications or any other factors that might increase cost for KTxR while hospitalized is important to improve our increasingly expensive healthcare economy.

The HCUP NIS has many strengths. It allows us to pull data from a large national sample size in order to accrue enough subjects into the transplant cohort to detect statistically significant data. HCUP NIS allows us to pull data from all different types of hospitals (rural vs. metropolitan, community vs. academic) that have been independently verified and audited. It also provides numerous variables regarding outcomes and patient characteristics that would be almost impossible to garner without the assistance of such a large database.

Despite these strengths, the main limitation of our report is the small sample size of the KTxR cohort. Unfortunately, this is a common theme in most transplant literature given the small portion of the population in the nation who undergo a transplant. Including other solid organ transplant recipients (liver, lung, heart, etc.) in our data would slightly increase our transplant recipient cohort size, but differences in immunosuppression regimen, insurance coverage, and patient characteristic would skew our data without adding much power. We predict that, over time, as the transplant population continues to increase in number with improved immunosuppression medications, that the sample size of KTxR would also increase if we repeated this project in the future, increasing the power of our data. Furthermore, to appropriately compare the transplant and non-transplant cohorts with such a disproportionate difference in sample size, most outcomes had to be measured in percentages to accurately reflect any differences between the two. Most literature involving transplant recipients often compare outcomes in transplant vs. non-transplant centers. However, we determined that the definition of a "transplant center" is very ambiguous, even classifying hospitals that have performed one transplant in the past decade as a "transplant center". We did not include transplant center as a part of our hospital characteristics for this reason. The cross-sectional nature of the HCUP NIS database only offers information regarding 30day mortality and in-hospital complications during a single hospital admission, limiting our abilities to determine long-term outcomes, tumor recurrence rate, and long-term mortality rate with this data. The clinical granularity of the HCUP NIS database, though it provides ICD-9 diagnosis code, does not provide information into tumor stage or type of thyroid cancer at time of diagnosis. It also prevents us from determining the extent of operative dissection performed at the time of thyroidectomy (including radical neck dissections, median sternotomies, etc.) The HCUP NIS database does not report severity of renal allograft dysfunction or immunosuppression regimen throughout the hospital stay, nor does it allow us to control for certain variables such as surgeon volume, hospital name, and socioeconomic status.

\section{Conclusion}

Our data should increase awareness of both surgeons and primary care physicians towards the development of thyroid cancer in their kidney transplant patients, promoting more aggressive screening and surgical treatment for thyroid cancer in this subset of patients. We also hope to dispel surgeons' fears towards performing thyroidectomies for thyroid cancer in transplant patients as transplant patients requiring chemotherapy and/or radiotherapy for any reason will have decreased graft survival and overall survival.

\section{Funding}

This research did not receive any specific grant from funding agencies in the public, commercial, or not-forprofit sectors.

\section{Disclosure of Interest}

The authors report no proprietary or commercial interest in any product mentioned or concept discussed in this article.

\section{References}

1. Soerjomataram I, Dikshit R, Eser S, Mathers C, Rebelo M, et al. (2013) GLOBOCAN 2012 v1.0, Cancer incidence and mortality worldwide: IARC CancerBase No. 11. World Health Organization.

2. Haugen BR, Alexander EK, Bible KC, Doherty GM, Mandel SJ, et al. (2016) 2015 American Thyroid Association Management Guidelines for Adult Patients with Thyroid Nodules and Differentiated Thyroid Cancer: The American Thyroid Association Guidelines Task Force on Thyroid Nodules and Differentiated Thyroid Cancer. Thyroid 26: 1-133.

3. Kitahara CM, Sosa JA (2016) The changing incidence of thyroid cancer. Nat Rev Endocrinol 12: 646-653.

4. Lim H, Devesa SS, Sosa JA, Check D, Kitahara CM (2017) Trends in Thyroid Cancer Incidence and Mortality in the United States, 1974-2013. JAMA 317: 1338-1348.

5. Engels E, Pfeiffer RM, Fraumeni JF Jr, Kasiske BL, Israni AK, et al. (2011) Spectrum of cancer risk among U.S. solid organ transplant recipients: the transplant cancer match study. JAMA 306: 1891-1901.

6. Hortland M, Arroyo Mühr LS, Storm H, Engholm G, Dillner $\mathrm{J}$, et al. (2017) Cancer risks after solid organ transplantation and after long-term dialysis. Int J Cancer 140: 1091-1101.

7. Karamchandani D, Arias-Amaya R, Donaldson N, Gilbert J, Schulte KM (2010) Thyroid cancer and renal transplantation: a meta-analysis. Endocr Relat Cancer 17: 159-167.

8. Keles $Y$, Tekin S, Duzenli M, Yuksel $Y$, Yücetin L, et al. 
(2015) Post-transplantation malignancy after kidney transplantation in Turkey. Transplantation Proceedings 47: 1418-1420.

9. Kitahara CM, Yanik EL, Ladenson PW, Hernandez BY, Lynch CF, et al. (2017) Risk of thyroid cancer among solid organ transplant recipients. Am J Transplant 17: 2911-2921.

10. Wisgerhof HC, van der Geest LG, de Fijter JW, Haasnoot GW, Claas FH, et al. (2011) Incidence of cancer in kidney-transplant recipients: a long-term cohort study in a single center. Cancer Epidemiol 35: 105-111.

11. Pond F, Serpell JW, Webster A (2005) Thyroid cancer in the renal transplant population: epidemiological study. ANZ J Surg 75: 106-109.

12. Lee J, Jeong JJ, Lee YS, Nam KH, Chang HS, et al. (2008) Incidence and clinical behavior of papillary thyroid carcinoma in renal allograft recipients: a single center experience. Transplant Proc 40: 3751-3754.

13. Hart A, Smith JM, Skeans MA, Gustafson SK, Stewart DE, et al. (2016) Kidney. Am J Transplant 16: 11-46.

14. 2018 USRDS Annual Data Report: Executive Summary.

15. de'Angelis N, Esposito F, Memeo R, Lizzi V, MartìnezPérez A, et al. (2016) Emergency abdominal surgery after solid organ transplantation: a systematic review. World $\mathrm{J}$ of Emerg Surg 11: 43.

16. Ueno P, Felipe C, Ferreira A, Cristelli M, Viana L, et al. (2017) Wound Healing Complications in Kidney Transplant Recipients Receiving Everolimus. Transplantation 101: 844-850.

17. DiBrito SR, Haugen CE, Holscher CM, Olorundare IO, Alimi $\mathrm{Y}$, et al. (2018) Complications, length of stay, and cost of cholecystectomy in kidney transplant recipients. Am J Surg 216: 694-698.

18. DiBrito SR, Alimi Y, Olorundare IO, Holscher CM, Haugen CE, et al. (2018) Outcomes following colorectal resection in kidney transplant recipients. J Gastrointest Surg 22: 16031610.

19. DiBrito SR, Olorundare IO, Holscher CM, Landazabal CS, Orandi BJ, et al. (2018) Surgical approach, cost, and complications of appendectomy in kidney transplant recipients. Clin Trans 32: 13245.

20. Introduction to the HCUP National Inpatient Sample (NIS) 2014.

21. Parsons HM, Habermann EB, Stain SC, Vickers SM, Al-Refaie WB (2012) What happens to racial and ethnic minorities after cancer surgery at American College of Surgeons National Surgical Quality Improvement Program hospitals? J Am Coll Surg 214: 539-547.

22. Zhang W, Lyman S, Boutin-Foster C, Parks ML, Pan TJ, et al. (2016) Racial and ethnic disparities in utilization rate, hospital volume, and perioperative outcomes after total knee arthroplasty. J Bone Joint Surg Am 98: 1243-1252.

23. Poudel A, Zhou JY, Story D, Li L (2018) Diabetes and Associated Cardiovascular Complications in American Indians/ Alaskan Natives: A Review of Risks and Prevention Strategies. J Diabetes Res 2018: 2742565.

24. Loehrer AP, Murthy SS, Song Z, Lubitz CC, James BC (2017) Association of Insurance Expansion with Surgical Management of Thyroid Cancer. JAMA Surg 152: 734-740.

25. Echanique KA, Govindan A, Mohamed OM, Sylvester M, Baredes S, et al. (2019) Age-related trends of patients undergoing thyroidectomy: analysis of US inpatient data from 2005 to 2013. Otolaryngol Head Neck Surg 160: 457464. 